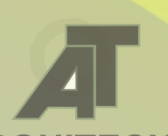

ARQUITECNO

\title{
POLÍTICAS DE GOBIERNO Y TRANSICIÓN TECNOLÓGICA
}

Eje 4: Hábitat e infraestructura

\author{
Lafalce Horacio R. ${ }^{1}$ \\ Larroque Luis A. ${ }^{2}$ \\ García Zúñiga Federico ${ }^{3}$ \\ Piñeyro María S. ${ }^{4}$ \\ Medina Darío R. ${ }^{5}$ \\ Oliva Jorge $A{ }^{6}$ \\ Perez Santiago M. A. ${ }^{7}$
}

1, 2, 3, 4, 5 y 6 Facultad de Arquitectura y Urbanismo - UNLP | Taller Vertical No 3 de Procesos Constructivos Lafalce - Larroque - García Zúñiga, Argentina, http/procesosconstructivos123.wordpress.com, hachelafa@yahoo.com.ar, federicogezeta@gmail.com

\section{RESUMEN}

Ya en el relato mitológico era visible que la humanidad se desarrollaría conforme la posibilidad de desarrollo tecnológico, tal como lo posibilita Prometeo cuando provee a los hombres el fuego y con él consecuentemente la posibilidad de ampliar el menú alimentario primitivo, y la metalurgia posteriormente. Entre estos dos acontecimientos se produce la más grande revolución de la humanidad, la revolución agrícola, que permitió la aparición de la urbe, la polis -y la política- a partir de la sedentarización.

Ahora bien, en el decurso posterior de los tiempos se sucedieron varios avances tecnológicos que acompañaron las necesidades emergentes de los distintos cambios culturales. Es necesario marcar que hacia el Siglo XV, Europa estaba saliendo de su aislamiento cultural gracias al contacto con el mundo árabe. Cambio que se aprecia claramente en el Renacimiento Italiano, con su espíritu cosmopolita y sincrético.

Pero el cristianismo, ignorando su origen oriental se romanogermaniza y se propone como modelo universal y con ello nace la empresa colonialista e imperialista que va a tener a América como uno de los destinos importantes, con una fundamental influencia en América Latina, dando nacimiento al eurocentrismo modelador de la impronta cultural, ocultando el problema de las identidades de los pueblos.

Al decir de Vasconcelos hay tres Américas, la indígena, la europea y la africana, además de las corrientes migratorias; italianas, españolas, judías, alemanas, sirio libanesas, etc. En la era de la globalización, de las comunicaciones en tiempo real y de la informática, aparecen luchas étnicas 
de fuerte intensidad, todo lo que nos hace pensar que a esta altura de los acontecimientos, no es posible pensar cambios culturales sin una visión universalista.

Ahora bien, en este contexto de cambios sí debemos pensar en la construcción del conocimiento en tecnología. Creemos que sin desmerecer todo lo pasado, aparece como un imperativo pensar en un devenir que se verá fuertemente condicionado por su relación con el medio natural y social, por lo que Producción, Ciencia y Tecnología pasa a ser el paradigma de una explosión de conocimientos que posibilitará el abordaje de situaciones que, aunque imaginadas, aún nos son desconocidas.

En el marco conceptual planteado, el objetivo de este trabajo es propender a que las políticas de gobierno -que deberían ser de estado- incorporen a las problemáticas del hábitat y la infraestructura, la transición tecnológica a los nuevos paradigmas planteados.

La gran mayoría de los países de Latinoamérica tienen, en estos temas, una semilla común, ser consumidores de tecnologías y modelos de los grandes centros de desarrollo tecnológico.

PALABRAS CLAVES: PRODUCCIÓN - CIENCIA - TECNOLOGÍA - TRANSICIÓN - POLÍTICA

\section{INTRODUCCIÓN}

Durante los años sesenta y en la década siguiente surgió en América Latina un movimiento intelectual orientado a buscar soluciones a los problemas del crecimiento económico e impulsar la modernización social dando un papel destacado a la ciencia y la tecnología en aquel proceso. Desde el punto de vista ideológico, aquel movimiento reflejaba en gran medida la visión del desarrollo inspirada por la CEPAL, si bien algunos autores se nutrieron de fuentes más claramente adscriptas a un pensamiento de izquierdas.

Entre los más destacados, Amílcar Herrera vinculaba el carácter marginal de la ciencia en la región con la dependencia de los centros de poder mundial y señalaba que la investigación científica tenía más relación con las necesidades internas del grupo social que las generaba, que con los requerimientos propios del desarrollo del país dependiente.

Otros, como Jorge Sábato y Máximo Halty creían en la capacidad local para alcanzar el desarrollo y regular los influjos de tecnología extranjera. Al mismo tiempo, en el propio núcleo de las comunidades científicas de los países de América Latina comenzó a manifestarse una actitud crítica respecto al modelo de desarrollo seguido hasta entonces con relación a la ciencia y la tecnología.

Oscar Varsavsky realizaba un cuestionamiento más radical que el de sus colegas, que se tradujo en la reivindicación del perfil del científico revolucionario y en la distinción entre la ciencia importada, copiada o generada localmente en función de demandas sociales, así como en la reflexión acerca del estilo de país que a cada una de ellas habría correspondido. No cualquier estilo científico es compatible con un estilo de sociedad determinada, afirmaba Varsavsky. 


\section{DESARROLLO}

El papel jugado por la Organización de los Estados Americanos (OEA) fue de gran importancia para el diseño de los instrumentos de política científica y tecnológica adoptados por América Latina. En forma convergente, también UNESCO contribuyó al primer diseño institucional de la política científica y tecnológica en la región. Del éxito de aquellos esfuerzos da cuenta el hecho de que los consejos de ciencia y tecnología fueran creados en forma mimética y casi simultánea en muchos países. Sin embargo, la economía real marchaba por otros carriles.

El proceso de industrialización sustitutiva propuesto por CEPAL como estrategia básica de desarrollo se nutrió fundamentalmente de tecnología transferida en forma incorporada a las grandes inversiones de capital. El resultado fue un nivel relativamente bajo en la capacidad tecnológica del sector productivo de los países latinoamericanos y una escasa demanda de conocimientos tecnológicos generados localmente. Una de sus consecuencias más evidentes fue el hecho de que la política inspirada, por lo demás, en el modelo que primaba la investigación básica estuviera escasamente vinculada con la eventual demanda tecnológica que pudiera provenir de los procesos económicos y sociales.

Resumiendo, sea a través de una jerarquía, de una relación funcional o de un vínculo presupuestario, se intenta asegurar el mínimo de coordinación necesaria para convertir políticas públicas en acciones burocráticas puntuales, coherentes con sus propósitos. Formalmente, la estructura y funcionamiento de cualquier burocracia pública prevé relaciones de interdependencia de estos tres tipos. El organigrama es un ejemplo de interdependencia jerárquica. El presupuesto, de interdependencia material. El "sistema de ciencia y tecnología", de interdependencia funcional. Se trata de construcciones ideales, donde se dibujan las redes circulatorias de autoridad, bienes e información que alimentan las relaciones intra-burocráticas. De aquí que la política y administración hayan sido vistas como esferas analítica e ideológicamente distinguibles, aunque empíricamente inseparables.

Ahora bien, independientemente de los avatares de las políticas y los políticos locales y regionales, debemos convenir que en el mundo se han seguido desarrollando tecnologías asociadas a la Investigación y Desarrollo, logrando avances significativos, de gran trascendencia para los procesos industriales y sociales.

Podríamos completar brevemente este panorama con las biotecnologías y su asombrosa capacidad para hacer o modificar productos, para provocar mutaciones en plantas y animales o para desarrollar microorganismos de uso específico mediante el estudio y la manipulación de los organismos vivos en el ámbito celular y molecular.

Lo mismo podríamos decir respecto de la revolución de los materiales novedosos para las nuevas tecnologías, la búsqueda de propiedades específicas, el diseño de materiales a voluntad que revolucionan incluso el concepto de investigación científica (asemejándola a la tecnología y a la ingeniería) y que permiten mejorar la eficiencia universal de los procesos, la resistencia a altas temperaturas, a los esfuerzos mecánicos, a la corrosión, a la mayor eficiencia energética y a la menor densidad. Los nuevos materiales extienden su uso a áreas distintas de las que provocaron su desarrollo e incluso, para el asombro de todos, vemos cómo van emergiendo tecnologías hasta ahora propias de la ciencia-ficción, como la de miniaturización, encarnadas en la nanotecnología. 
Ahora bien, el destino de nuestras sociedades está ineludiblemente ligado a las decisiones políticas que se tomen. Ciertamente, no estamos ante un incremento del modelo industrial de posguerra, sino ante una nueva realidad.

Una realidad en la que no es fácil acceder a posiciones mejores, pero aún así tenemos una oportunidad. Los cambios generan nuevas posibilidades..." "cada revolución tecnológica es un "huracán de destrucción creativa" que transforma, destruye y renueva el aparato productivo mundial»"

Por eso, algunos de los principales retos a afrontar como habitantes de la aldea global, posicionados en América Latina, son: cómo convertir información en conocimiento útil, y cómo inducir procesos de aprendizaje social del conocimiento.

La gran mayoría de los países del llamado Tercer Mundo no transitaron un camino similar al de Europa, Estados Unidos y Japón. Éste fue el último en llegar, y por ello mismo, ejemplo señero y notable en la adopción de la ciencia y la tecnología como base del desarrollo y creador de esperanzas en el mundo en desarrollo de entonces. De hecho, algunas sociedades asiáticas lo adoptaron y esto permitió el renacer de un «optimismo histórico»: sí era posible entrar al círculo privilegiado.

Hasta hace pocas décadas estaba por fuera del horizonte de los países del Tercer Mundo alentar estas actividades para propulsar el desarrollo económico.

De modo similar a como se tenían políticas educativas, de comercio exterior y agrícolas o industriales, nacieron en los años sesenta políticas de Ciencia y Tecnología, para trazar pautas de fomento y organización de esta compleja actividad.

Según la observación de Francisco Sagasti, fue justamente el éxito de la industrialización japonesa el que despertó grandes inquietudes sobre el papel que jugaron la tecnología y la educación en dicho proceso; y sirvió de inspiración en la medida en que los japoneses habían hecho su desarrollo prestando y transfiriendo tecnología más que desarrollándola originalmente.

La atracción de los países en desarrollo hacia los problemas de la política científica y tecnológica "prosigue Sagasti», se deben en buena medida al fracaso relativo que han tenido los procesos de industrialización en la post guerra.

La importancia que tomaron la ciencia y la tecnología en el mundo industrializado (fenómeno que registró e impulsó la OCDE), fue un factor que despertó gran interés en los países en desarrollo. La difusión realizada por los organismos internacionales de esta nueva perspectiva del crecimiento contribuyó a la adopción de medidas públicas relacionadas con el fomento de la ciencia y la tecnología.

En un primer momento, esta preocupación se orientó tanto a identificar instituciones, capacidades y recursos de ciencia y tecnología presentes en los países industrializados y ausentes en los subdesarrollados, como a obtener información sobre cómo lo hacían. Se trató de ver cómo funcionaban allí la I+D, las instituciones de educación superior y los organismos de apoyo a la ciencia. 


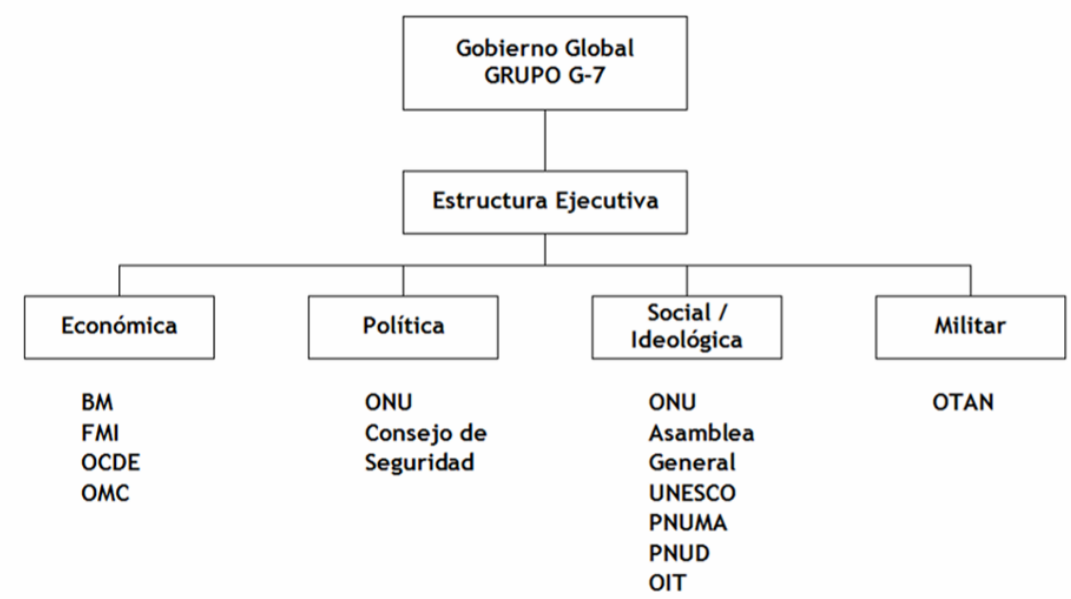

Fig. 1: El gobierno global.

Elaboración propia a partir de Chomsky y Dieterich. La sociedad global. Educación, mercado y democracia, 1996.

Ciertos documentos aportaron sus luces en el escenario latinoamericano y sentaron las bases conceptuales para orientar las políticas en estos dominios.

En 1969, Jorge Sábato y Natalio Botana publicaron un documento de gran influencia: La Ciencia y la Tecnología en el desarrollo futuro de América Latina. En él examinan con profundidad las tareas que corresponden al Estado, a la comunidad científica y al sector empresarial, ocupándose de señalar también las relaciones coherentes que estos deben construir para incorporar al desarrollo de los países latinoamericanos una variable de semejante poder.

Sin Ciencia y Tecnología, advertían Sábato y Botana de manera premonitoria, las naciones latinoamericanas se quedarían sin soberanía, solo con sus símbolos, las banderas y los himnos, pero sin viabilidad histórica.

El modelo latinoamericano de industrialización de tipo proteccionista y por sustitución de importaciones, engendró su propio estilo tecnológico y esto determinó implícitamente, entre otras cuestiones, el uso de insumos tecnológicos importados en detrimento de los de origen local. Otros países, en particular los asiáticos, siguieron políticas de exportaciones y de conexión con los mercados mundiales, lo que los condujo a otro tipo de perfil tecnológico, mucho más fuerte.

\section{CONCLUSIÓN}

Desde los tiempos de la antigüedad clásica, el supremo ideal de las utopías políticas consistió en lograr que la capacidad racional y deliberativa de gobiernos y gobernantes y la pacífica fuerza de la ilustración, guiaran a los hombres hacia empresas cooperativas que materializan el bien común. Pero el marco normativo y axiológico del comportamiento humano siempre ha reconocido múltiples estímulos, entre los cuales la búsqueda del interés colectivo no ha sido precisamente el dominante. No obstante, la actividad humana, desarrollada a través de organizaciones, exige un continuo compromiso entre los fines individuales y los fines colectivos que dan sentido a la acción organizacional.

Por su parte el gobierno argentino en su Plan Estratégico de Ciencia, Tecnología e Innovación "Bicentenario" (2006-2010)", estable que las políticas de CTI "pueden y deben contribuir a un mejoramiento de las condiciones de desarrollo sustentable e inclusión social con vistas la 
'resolución de problemas y el aprovechamiento de oportunidades en articulaciones socioproductivas, en núcleos socio productivos estratégicos que tengan alto impacto económico, tecnológico o social. Asimismo, se plantea impulsar "la innovación productiva inclusiva y sustentable".

No dejamos de notar que el progresivo deterioro de los estamentos directivos de los organismos públicos se debe más a conductas políticas que a políticas tecnológicas, puesto que en los últimos decenios vemos que -más allá de que se desarrollaran algunas iniciativas casi todas sin éxito desde principios de los años '80 del siglo pasado- en las áreas técnicas de la Administración Publica los puestos directivos ya casi no son ocupados por personal de carrera, sino que en la mayoría de los casos lo son por personas funcionales a los intereses políticos de turno logrando por lo tanto una difícil sino imposible coexistencia entre Investigación y Administración, que, aunque parezca descabellado hubo en épocas no tan lejanas equipos de trabajo en los ministerios que se preocupaban de investigar y formar en apreciaciones de nuevos sistemas y técnicas.

\begin{tabular}{|c|c|c|c|}
\hline Iniciativa & Década de inicio & $\begin{array}{c}\text { Período de } \\
\text { implementación }\end{array}$ & Tipo de modelo \\
\hline $\begin{array}{c}\text { Administradores } \\
\text { Gubernamentales }\end{array}$ & $80^{\prime}$ & Tres años (3) & Carrera \\
\hline SINAPA & $90^{\prime}$ & Nueve años (9) & Mixto \\
\hline $\begin{array}{c}\text { Economistas de } \\
\text { Gobierno }\end{array}$ & $90^{\prime}$ & Seis años (6) & $\begin{array}{c}\text { Carrera } \\
\text { especializado }\end{array}$ \\
\hline Gerentes Públicos & 2000 & 5 meses & $\begin{array}{c}\text { Sistema de } \\
\text { empleo o } \\
\text { posiciones }\end{array}$ \\
\hline SINEP & 2008 & Sin & Mixto \\
\hline
\end{tabular}

Fig. 2: Iniciativas de Alta Dirección Pública 1983-2008.

En Zuvanic y Diéguez, 2016.

En el trabajo citado se resume claramente el problema, donde se establecen dos aspectos que confirman esta visión: sólo el $5 \%$ de los cargos de la Alta Dirección Pública son asignados por Concurso y la mayoría son designados bajo el formato de "asignación transitoria de funciones superiores". Por otro lado, si bien los niveles de formación y calificación son altos (un $72 \%$ del segmento de los directivos públicos posee título de grado y/o posgrado), los mismos no están necesariamente preparados para el cargo, muchas veces por no coincidir la especialidad profesional con el cargo ejercido, y sobre todo al no existir casi alternativas de formación en la carrera de la alta administración pública.

Pero sabiendo quiénes somos y donde estamos, es que pensamos que deberían formarse en la órbita de la Administración Publica, Organismos de investigación que, orientados al perfeccionamiento de los sistemas y tecnologías en uso, como así también la posibilidad de diseñar y/o crear aquellas que resulten pertinentes a la satisfacción de las demandas que del alto cúmulo de necesidades sociales emerjan.

No ignoramos que todo lo antes dicho posee una alta carga simbólica que permite múltiples lecturas, pero ante todo somos Arquitectos, Docentes y HABITANTES DE UNA REALIDAD QUE 
SE NOS OCURRE CADA VEZ MAS DIFICIL..." PERO QUIZA DE AHÍ SE TENGA UNA OPORTUNIDAD SUPERADORA"

\section{BIBLIOGRAFÍA}

Chomsky, N y Dieterich, H. La sociedad global. Educación, mercado y democracia. Santiago de Chile: LOM Ediciones, 1996.

Oszlak, O. Politicas públicas y regímenes políticos: reflexiones a partir de algunas experiencias latinoamericanas. Conferência Internacional LALICS 2013 "Sistemas Nacionais de Inovação e Políticas de CTI para um Desenvolvimento Inclusivo e Sustentável". 11 e 12 de Novembro, 2013.

Pérez, C. Cambio tecnológico y oportunidades de desarrollo como blanco móvil, en. Sagasti, F., Crisis y desafío: ciencia y tecnología en el futuro de América Latina, en Comercio Exterior (1988), vol. $38, n^{\circ} 12$. Id., La ciencia y la tecnología durante el decenio de los ochenta, en Comercio Exterior (1988), vol. 37, nº 12.

Piñón, F. Temas de Iberoamérica. Globalización, Ciencia y Tecnología

Sábato, J. A. y Botana, N., La ciencia y la tecnología en el desarrollo futuro de América Latina, en Revista de la Integración, INTAL, Buenos Aires 1968, Año 1, n. 3, pp. 15-36.

Sagasti, F. y Arévalo, G., América Latina en el nuevo orden mundial fracturado: perspectivas y estrategias, en Comercio Exterior (1992), 42(12).

Zuvanic, L y Diéguez, G. El juego de la oca y la Alta Dirección Pública en Argentina. Desafíos y propuestas para construir directivos públicos idóneos. CIPPEC (Centro de Implementación de Políticas Públicas para la Equidad y el Crecimiento). Área de Estado y Gobierno. Programa de Gestión Pública. Documento de Políticas Públicas. Diciembre de 2016. 